\title{
Repeated Exposure to Ketamine in Adolescent Rats Results in Persistent Anxiety in the Adulthood
}

\author{
Manuela Amorim ${ }^{1-4}$, Joana Bravo ${ }^{1-3}$, Ana Isabel Silva ${ }^{1,2,5}$, Cecília Juliana Alves ${ }^{1,4,6}$, Pedro RR Monteiro ${ }^{4}$, Ana \\ Magalhães $^{1,2}$ and Teresa Summavielle $e^{1,2,4 *}$ \\ ${ }^{1}$ Addiction Biology Group, Instituto de Investigação e Inovação em Saúde (I3S), Universidade do Porto, Rua Alfredo Allen 208, \\ 4200-135 Porto, Portugal \\ ${ }^{2}$ Addiction Biology Group, IBMC-Instituto de Biologia Molecular e Celular, Universidade do Porto, Portugal \\ ${ }^{3}$ Instituto de Ciências Biomédicas de Abel Salazar, Universidade do Porto, Portugal \\ ${ }^{4}$ Escola Superior de Saúde-Instituto Politécnico do Porto (ESS-IPP), Portugal \\ ${ }^{5}$ Faculdade de Medicina da Universidade do Porto (FMUP), Portugal

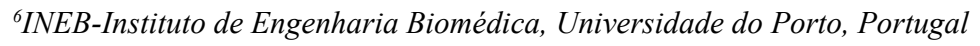 \\ Address correspondence to Teresa Summavielle, tsummavi@ibmc.up.pt
}

Received 19 July 2018; Revised 30 August 2018; Accepted 06 September 2018

Copyright $($ C 2018 Manuela Amorim et al. This is an open access article distributed under the terms of the Creative Commons Attribution License, which permits unrestricted use, distribution, and reproduction in any medium, provided the original work is properly cited.

\begin{abstract}
Background. Adolescent development of the prefrontal cortex (PFC) is accompanied by important changes in glutamatergic, GABAergic and dopaminergic circuitries, susceptible to modulation by N-methyl-D-aspartate receptors (NMDAR) antagonists. Repeated ketamine was associated with social and memory deficits, but other relevant factors, such as anxiety, were not sufficiently addressed. The present study aimed to examine the behavioral and molecular consequences of repeated exposure to ketamine with a particular focus in anxiety. Methods. We treated male adolescent Wistar rats, starting postnatal day (PND) 35, with ketamine $(30 \mathrm{mg} / \mathrm{kg}$, i.p, 7 days). Behavioral evaluation was conducted in the adulthood (PND $60)$. The elevated plus maze (EPM) and open field tests were used to evaluate anxiety and locomotion, while sociability and novelty recognition were assessed through the novel object recognition (NOR) and the sociability and social novelty tests. At the end of the behavioral evaluation, brains were dissected and the prefrontal cortex used for biochemical evaluation. Results. Analysis of the elevated plus maze (EPM) data revealed a ketamine-induced anxiety-like profile, corroborated by the open field data. Ketaminetreated rats also failed to increase contact time with a conspecific in the social affiliation test and with an unknown rat in the novelty preference test. At the molecular level, frontal expression levels of tyrosine hydroxylase were found decreased. Conclusion. Altogether, these results show that repeated ketamine-exposure in the adolescent may result in long-term anxiety.
\end{abstract}

Keywords ketamine; NMDAR; adolescent; anxiety; schizophrenia; prefrontal cortex

\section{Introduction}

Non-competitive N-methyl-D-aspartate receptor (NMDAR) antagonists, such as phencyclidine (PCP), dizocilpine (MK-801) and ketamine are known to modulate the prefrontal function and hence often used in rat and mouse models to mimic schizophrenia-like symptoms [1]. Initial evidence for these models comes from the recreational use of PCP that leads to delusion, hallucination and other psychotic behaviour in healthy subjects [2]. Importantly, different administration schedules were seen to result in substantially diverse outcomes as reviewed by Jones et al. [3] Typically, poor social interaction [4], anhedonia [5], and deficits in learning, memory or attention [3] were reported in NMDA antagonist models. These changes were associated with frontal dopaminergic hypofunction [6], abnormal cortical levels of glutamate [7] and decreased parvalbumin in the prefrontal cortex (PFC) and hippocampus $[5,8]$. Monoaminergic receptors were also affected in several brain regions $[9,10]$. Preclinical studies also report that sub-anesthetic doses of ketamine in adult rats may result in other schizophrenia-associated features such as nitrergic and GABAergic alterations in the hippocampus [11]; long-term increase of type-2 dopamine receptor 
$\left(\mathrm{DR}_{2}\right)$ binding in the hippocampus; decreased glutamate receptor binding in the PFC [12]; increased cortical levels of glutamate [13]; and a steady decline of both gamma and theta hippocampal oscillations correlated to reduced parvalbumin interneurons [14].

Because the use of NMDAR antagonists and other psychoactive substances $[15,16]$ in adolescence is frequently associated with the development of schizophrenic-like symptoms and psychosis, addressing exposure to ketamine during this developmental period could have increased etiological relevance [17]. Importantly, anxiety and poor sociability are often present at the onset of drug use/abuse, and may precede the arrival of psychotic surges $[18,19]$. In previous studies, chronic ketamine in adolescent rats resulted in impaired social behavior and memory deficits after 7 days of 25 $\mathrm{mg} / \mathrm{kg}$ [20], while other authors reported memory deficits after 7 days of $80 \mathrm{mg} / \mathrm{kg}$ of ketamine, but not after 30 $\mathrm{mg} / \mathrm{kg}$ [21]. In a different study adolescent rats exposed to ketamine $(30 \mathrm{mg} / \mathrm{kg}, 5$ days) displayed impaired pre-pulse inhibition and compromised memory [22]. However, chronic ketamine $(20 \mathrm{mg} / \mathrm{kg}$, twice a day) in the adolescent rat was also reported to endure resilience and presumed useful as an antidepressant [23]. Of note, repeated administration of $10 \mathrm{mg} / \mathrm{kg}$ dose was recently shown to elicit condition place preference in male rats [24]. In previous studies, behavioral evaluation was usually focused on domains related to attention, working memory, problem solving and social deficits [17], while anxiety-like symptoms were mostly neglected [3].

Here, aiming to better understand the effect of repeated ketamine in adolescent rats in anxiety and sociability, we exposed male adolescent Wistar rats to daily doses of $30 \mathrm{mg} / \mathrm{kg}$ of ketamine for 7 days. Evaluations were conducted at the onset of adulthood.

\section{Methods}

\subsection{Animals}

A total of 10 males born from primiparous threemonth old SPF Wistar females were used in this study (acquired from Charles River Laboratories, Barcelona, Spain). Adolescent females were excluded because the estrous cycle was recently demonstrated to interfere with anxiety levels in Wistar rats [25]. Animals were kept under stable conditions $\left(20-22^{\circ} \mathrm{C}, 60 \%\right.$ humidity) in an inverted 12-hour light/dark cycle, with water and food supplied ad libitum. Cylindrical card tubes and soft paper were made available. All procedures used were approved by local ethical committee and by the Portuguese Agency for Animal Welfare, general board of Veterinary Medicine (ref. 0421/000/000/2013), in compliance with the 2010 European Community Council Directive (2010/63/UE) and the ARRIVE guidelines. Procedures involving animals were conducted by FELASA C graded researchers and all efforts were made to minimize stress and discomfort.
At postnatal (PND) 30, adolescent male rats from different litters were randomly assigned to an experimental group and housed in pairs. From PND35, one rat per cage received a daily intraperitoneal administration of $30 \mathrm{mg}$ of ketamine $/ \mathrm{kg}$ of body weight, in a volume of $1 \mathrm{~mL} / \mathrm{kg}$, while the other rat received isovolumetric saline $(\mathrm{NaCl} 0.9 \% \mathrm{w} / \mathrm{v})$. Doses were administered one hour before the end of the dark period for seven consecutive days following previously reported schedules [11,12]. Ketamine (Imalgene1000 ${ }^{\circledR}, 100 \mathrm{mg} /$ $\mathrm{ml}$, racemic formulation) was obtained from Merial Portuguesa (Rio de Mouro, Portugal). Weight gain was controlled during the administration period. Behavioral assessment started at PND60, a time-point that matches the beginning of adulthood [26]. Rats were killed after the last behavioral evaluation by decapitation and brains were collected, dissected on ice and kept at $-80^{\circ} \mathrm{C}$ until processed.

\subsection{Behavioral testing}

Behavioral testing were performed in the adulthood (PND60). All tests were conducted in the dark phase of the light cycle, in a room adjacent to the maintenance area. All apparatus were thoroughly cleaned with neutral soap after each session. All evaluations were conducted in a blind manner.

\subsection{Elevated plus-maze (EPM)}

Rats were allowed to explore the maze individually for 5 minutes. Evaluated parameters: i) number of entries and time spent in open or closed arms and central platform; ii) self-grooming; iii) exploratory behavior; and iv) head dipping (HD) (head and shoulders over the edge of the maze) [27]. HD was considered protected (PHD) if the posterior paws were in the closed arms or central area, and unprotected if all paws were on open arms. Distal HD (DHD) took place on the distal one-third of the open arm. Video data were analyzed using the Observer 5 software (Noldus Information Technology, Wageningen, The Netherlands).

\subsection{Open field}

Each rat was placed in the center of an arena and allowed to move freely for $10 \mathrm{~m}$. The distance travelled, peripheral activity or thigmotatic behavior (locomotion along the walls) and center activity (locomotion in the central area) were automatically obtained through video tracking (Smart Video Tracking Software v 2.5, Panlab).

\subsection{Novel object recognition (NOR)}

The NOR test was performed as previously described [28]. During the habituation phase rats explored the apparatus for $10 \mathrm{~min}$. The acquisition/sample phase started a day after by placing each rat in the apparatus with two identical objects (familiar object) for $10 \mathrm{~min}$ (Figure 1A, 2A). After 24 hours (inter-trial interval), the retention/choice session was performed. In this phase, a novel object and a copy of the familiar object were used. 
Rats were again allowed to explore the objects for 10 min (Figure 3A). Exploration was defined as follows: rat nose touching or directed towards the object at a distance shorter than $2 \mathrm{~cm} \mathrm{[29].} \mathrm{The} \mathrm{discrimination} \mathrm{index} \mathrm{(DI)} \mathrm{was}$ calculated as the ratio $\mathrm{TN} /(\mathrm{TN}+\mathrm{TF})[\mathrm{TN}=$ time exploring the novel $(\mathrm{N})$ object; $\mathrm{TF}=$ time exploring the familiar $(\mathrm{F})$ object][29].

\subsection{Sociability and social novelty}

The apparatus contained three-chambers separated by clear plexiglas walls with doors. Each tested rat was placed in the central chamber and allowed to habituate for $5 \mathrm{~min}$. Testing was conducted as previously described [30]. During the social affiliation test, each rat was allowed to choose between the chamber with an unfamiliar rat (restrained in a containment cup, stranger I) or an empty cup (Figure 2D). The time spent around each cup was registered. For the social preference test, an additional rat (stranger II) was placed in the empty chamber (within the containment cup, Figure 2F). The experimental rat was allowed to explore the apparatus and the time spent around each conspecific was registered (contact time). Both tests lasted $10 \mathrm{~min}$. Video data were analyzed using the Observer 5 software (Noldus Information Technology).

2.7 Quantitative real-time polymerase chain reaction (qRT-PCR)

Since the prefrontal cortex is a brain region highly affected by ketamine [31,32], total RNA was isolated from the prefrontal cortex tissue using the iTaqTM Universal kit (Invitrogen, Carlsbad, CA, USA). RNA quality was confirmed in an agarose gel and RNA concentration determined in a NanoDrop ${ }^{\mathrm{TM}} 1000$ (Thermo Fisher Scientific, Wilmington, Delaware, USA). Synthesis of cDNA was performed using the NZY First-Strand cDNA synthesis kit (Invitrogen) and $1 \mu \mathrm{g}$ of RNA was used. The qRT-PCR reactions for the different genes, tyrosine hydroxylase (TH) and dopamine receptor 1 (DR1) and DR2, were performed on the iQ5 Multicolor Real-time PCR Detection System from Bio-Rad Laboratories (Hercules, CA, USA), using the iQTM SYBR ${ }^{\circledR}$ Green Supermix (Bio-Rad Laboratories, Hercules, CA, USA). mRNA quantification was performed by comparative threshold cycle quantification ( $\Delta \mathrm{Ct}$ method) using glyceraldahyde-3-phosphatedehydrogenase (GAPDH) as a reference gene. The primers used were obtained from Sigma (St. Louis, MO): TH sense primer: 5'-GGC TTC TCT GAC CAG GTG TAT C-3'; TH antisense primer: 5'-CAA TCT CTT CCG CTG TGT ATT CC-3'; D1R sense primer: 5'-ACT CTG TCT GTC CTT ATA TCC TCC-3'; D1R antisense primer - 5'-GTT GTC ATC CTC GGT GTC C 3'; D2R sense primer: 5'-CAA CAA TAC AGA CCA GAA TGC GTG-3'; D2R antisense primer 5'-CAG CAG AGT GAC GAT GAA GG 3'; GAPDH sense primer: 5'-TTC AAC GGC ACA GTC AAG g-3'; GAPDH antisense primer: 5'-CTC AGC ACC AGC ATC ACC-3'.

\subsection{Western blot}

Brain tissue from the prefrontal cortex was also homogenized in lysis buffer $(50 \mathrm{mM}$ Tris- $\mathrm{HCl}, 2 \mathrm{mM}$ EDTA, $150 \mathrm{mM} \mathrm{NaCl}, 0.5 \%$ NP-40, 1\% Triton X-100, supplemented with 1:500 Protease Inhibitor Cocktail, Sigma, St. Louis, MO, USA) and centrifuged at 16000 $\mathrm{g}$ for $10 \mathrm{~min}$ at $4^{\circ} \mathrm{C}$. Total protein concentration was estimated in the supernatant by the Bradford method (BioRad Protein Assay, Munich, Germany). Protein samples were resolved by SDS-PAGE on $12 \%$ polyacrylamide gels or by tricine-SDS-PAGE for parvalbumin, and transferred to PVDF membranes by electroblotting. Membranes were blocked with BSA 5\% and incubated with one of the following: Rabbit anti-PSD-95 (Cell Signaling Technology, \#3450, 1:1000), goat antiGAD-67 (Santa Cruz Biotechnology, sc-7512, 1:300), mouse anti-NMDAR1 (Millipore, MAB363, 1:500) and rabbit anti-parvalbumin (Abcam, ab11427, $0.3 \mu \mathrm{g}$ / $\mathrm{ml}$ ); or blocked with dry milk and incubated with rabbit anti-VGLUT (Synaptic Systems, 135 303, 1:5000). A mouse anti-GAPDH (Hytest, \#5G4, 1:100000) primary antibody was used in all membranes for loading control. The secondary antibody HRP (horseradish peroxidase)conjugated was used for $1 \mathrm{~h}$. Chemiluminescent signal detection was achieved using the Immun-Star HRP kit (Bio-Rad Laboratories). A GS800 densitometer (BioRad Laboratories) and quantity one 1-D analysis software (v4.6, Bio-Rad) were used for analysis.

\subsection{Statistical analyses}

Weight evolution through the experimental period was analyzed using two-way ANOVA (Time $\mathrm{x}$ Treatment) with repeated measures followed by the Sidak multiple comparisons test. Unpaired Student's two-tailed t-test was used to compare ketamine treated animals with the control group in the EPM, open field and NOR tests, and also for molecular data. Two-way ANOVA (Treatment $x$ Social/Novelty preference) followed by the Sidak multiple comparisons test was used to analyze data from social and novelty preference tests. Statistical significance was set at $P<0.05$. Data were presented as mean $\pm \mathrm{SEM}$. All tests were conducted using the GraphPad-Prism software. version 7.00 for Mac Os X (GraphPad Software, Inc., San Diego, CA). The G*Power 3.1 software [33] was used to ensure that the achieved power in each test was above 0.95 . Critical $\mathrm{t}$-value were also considered.

\section{Results}

\subsection{Ketamine exposure did not affect weight evolution}

Analysis of body weight revealed no differences between ketamine-treated rats and control rats for all examined time points (Sup. Figure 1, $F(7,56)=2.527$, $P=0.1506)$. Of note, no differences were observed between the initial body weight in both groups $\left(P_{(\mathrm{U}=11)}=0.8175\right)$. Of note, no signs of discomfort were observed during the entire experimental period. 


\subsection{Ketamine induced anxiety-like behavior}

To assess the effect of ketamine in anxiety, rats were tested in the EPM at PND60. Ketamine-treated rats showed decreased time spent in the center platform $\left(P_{(\mathrm{t}=3.04, \mathrm{dF}=8)}=0.0161\right.$, Figure 1A) and increased time in closed arms $\left(P_{(\mathrm{t}=2.436 \mathrm{dF}=8)}=0.0408\right.$, Figure 1A $)$, which was concomitant with reduced frequency of entries in open arms for the ketamine group $\left(P_{(\mathrm{t}=2.554, \mathrm{dF}=8)}=0.0340\right.$, Figure 1B). No differences were observed in total arm entries, a measure of global activity (Figure 1C, $P_{(\mathrm{t}=1,824}$ $\mathrm{dF}=8)=0.1056)$. Environment exploratory scanning was higher in ketamine-treated rats (Figure 1D, $P_{(\mathrm{t}=3.041}$ $\left.P_{(\mathrm{t}=3.041, \mathrm{dF}=8)}=0.0161\right)$. The time spent in DHD, a measure of exploration, was decreased in ketamine-exposed rats $\left(P_{(\mathrm{U}=2.5)}=0.0476\right.$, Figure 1E).

The open field test was conducted 2 days after the EPM. In this test, the percent of time spent in the peripheral area was increased in ketamine-treated rats, while the percent of time in the center was decreased for the same group $\left(P_{(\mathrm{t}=2.519, \mathrm{dF}=8)}=0.0359\right.$ for both, Figure $1 \mathrm{~F})$. There was no significant difference in the total distance traveled by both groups $\left(P={ }_{(\mathrm{t}=1.092}, \mathrm{dF}=8\right)=0.3067$, Figure1 G).

3.3 Ketamine decreased the exploration and social novelty preference

The NOR was used to evaluate the impact of the selected ketamine administration schedule in novelty recognition. As plotted in Figure 2B, both groups were able to discriminate between the novel and the familiar object as traduced by the discrimination index $\left(P_{(\mathrm{t}=0.2982, \mathrm{dF}=8)}=0.7731\right)$. However, the total exploration time was reduced in the ketamine treated group $\left(P_{(\mathrm{t}=3.074}\right.$, $\mathrm{dF}_{\mathrm{B}}=0.0153$, Figure 2C).

In the social affiliation test, rats were allowed to choose between contact with an empty containment cup and a restrained unfamiliar rat (stranger I) (Figure 2D). Analysis of the time spent around each cup revealed a significant effect of social affiliation $\left(\mathrm{F}_{(1,16}\right)=12.06$, $P=0.0031$ ). Further analysis by Sidak's multiple comparison showed a significant increase in contact time in the compartment holding a conspecific for the control group ( $P=0.0138)$ but not for ketamine $(P=0.1688$, Figure 2E).

To assess social novelty preference, a second unfamiliar rat was placed in the empty cup. Preference between the familiar rat (stranger I) and the unknown rat (stranger II) was evaluated through the time the focal rat spent in contact with each conspecific (Figure 2F). A two-way ANOVA for "contact time" revealed an effect of social novelty $\left(\mathrm{F}_{(1,16)=} 11.67, P=0.0035\right)$. A Sidak's post-hoc test showed increased contact time with the novel subject (stranger II) in the control group $(P=0.0306$, Figure $2 \mathrm{G})$, but not in the ketamine group
$(P=0.0976$, Figure 2G).

3.4 Ketamine affected dopaminergic but not glutamatergic and GABAergic markers

Prefrontal glutamatergic pyramidal neurons are modulated by parvalbumin expressing GABAergic interneurons, which were reported to produce reduced amounts of GAD67 [34]. We assessed the expression levels of several proteins likely to be affected within these circuitries (Figure 3A-F). For pyramidal glutamatergic neurons we used western blot to analyze the levels of VGLUT1 (a glutamate transporter), PSD95 (a marker of glutamatergic synapses) and NMDAR1 (a glutamate receptor). No significant differences were seen when comparing frontal extracts from ketamine exposed with those of control rats $\left(P_{(\mathrm{U}=8)}=0.4206\right.$, Figure $3 \mathrm{~A} ; P_{(\mathrm{U}=7.5)}=0.3413$, Figure $3 \mathrm{~B}$; and $P_{(\mathrm{U}=9)}=0.5476$, Figure 3C). Parvalbumin and GAD67, markers of GABAergic interneurons were also not affected by ketamine $\left(P_{(\mathrm{t}=1.329}\right.$, $\mathrm{dF}=8)=0.2204$, Figure 3D; and $P_{(\mathrm{t}=0.2558, \mathrm{dF}=8)}=0.8045$, Figure 3E).

The mRNA levels of the dopamine receptor (DR)1 and DR2 were not altered by ketamine exposure $\left(P_{(\mathrm{t}=0.5976}\right.$, $\mathrm{dF}_{\mathrm{d}=6)}=0.5720$, Figure $3 \mathrm{H} ;$ and $P_{(\mathrm{t}=1.248, \mathrm{dF}=6)}=0.2586$, Figure 3I). The mRNA expression levels of tyrosine hydroxylase $(\mathrm{TH})$, however, were significantly reduced in the ketamine treated rats $\left(P_{(\mathrm{t}=3.63, \mathrm{dF}=6)}=0.0110\right.$, Figure $3 \mathrm{~J})$, which may result in decreased dopamine synthesis.

\section{Discussion}

The present administration schedule aimed at further characterizing the persistent effects of a repeated administration of ketamine during the adolescent period. Our results show that repeated adolescent exposure to ketamine lead to a shift towards increased anxiety-like states, which was concomitant with impaired social and novelty preference. At the molecular level we found decreased $\mathrm{TH}$ gene expression in the PFC, while the frontal levels of parvalbumin, GAD67 and NMDAR, hallmarks of frontal GABAergic and glutamatergic function, were not significantly affected by ketamine.

We started our evaluation by conducting an EPM test. Our results consistently showed a ketamineinduced anxiety-like profile. Decreased entries in open arms, decreased time in central platform, decreased head dipping and augmented environment scanning are well-recognized anxiogenic features [35]. Blockade of NMDAR through developmental periods was previously seen to cause persistent anxiety in the adulthood [36]. In contrast, a former study using a similar exposure to ketamine found decreased anxiety in the EPM [23]. However, in that work the EPM was conducted after a locomotion evaluation, which may have affected the EPM performance, since previous experience is known to affect anxiety-like responses [37]. Of note, agitation, anxiety 
A

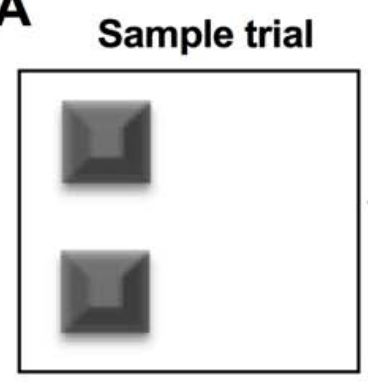

Familiar object

D

Social affiliation (10min)

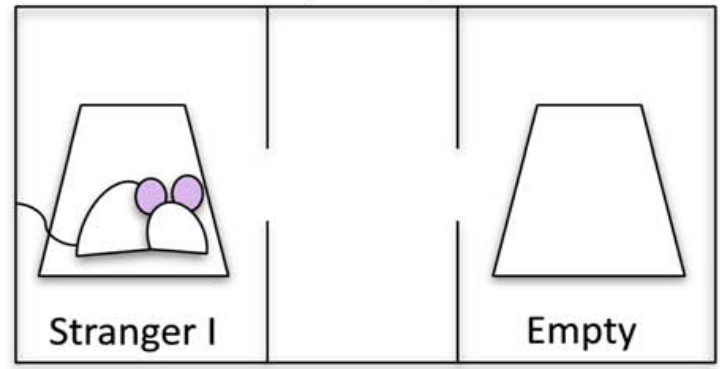

F Social Novelty/preference $(10 \mathrm{~min})$

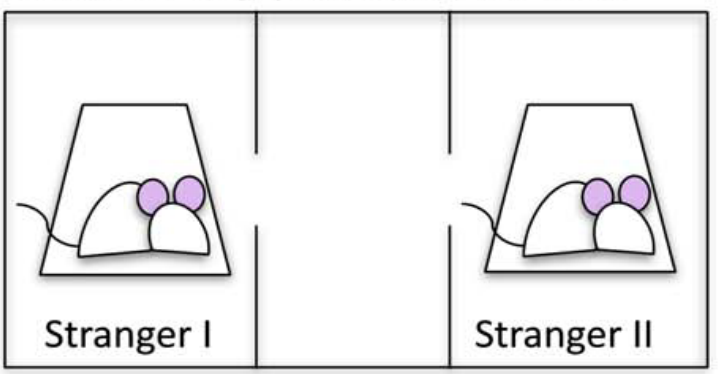

B

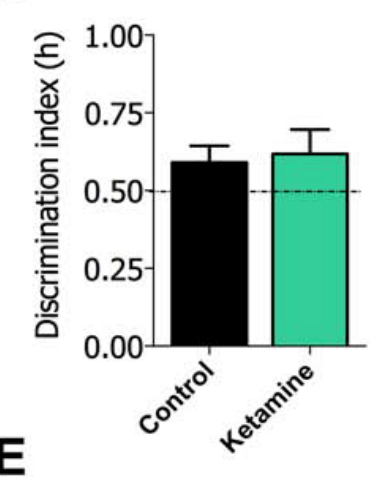

E

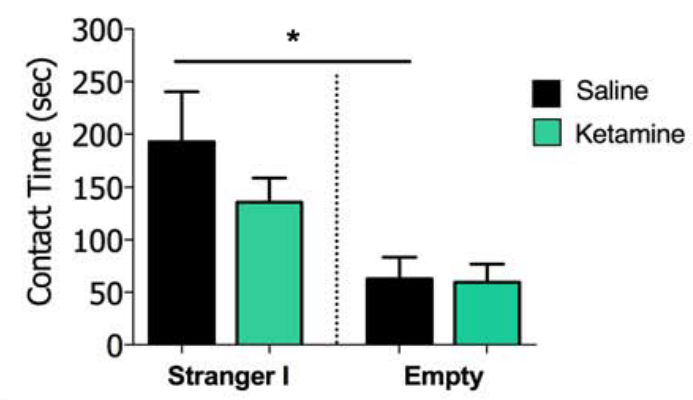

G

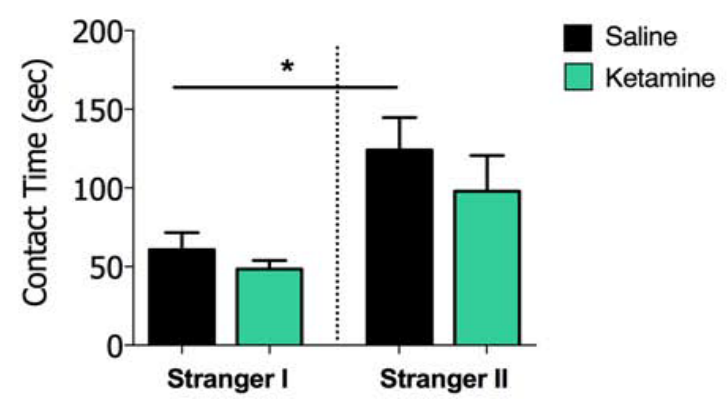

Figure 2: Ketamine decreased exploration and novelty preference (A) Schematic diagram of sample (acquisition) and choice phase conditions in the NOR test. The trial phase was conducted $24 \mathrm{~h}$ after the sample phase. (B) No significant differences were observed for the discrimination index between ketamine exposed and control rats, calculated as the ratio $\mathrm{TN} /(\mathrm{TN}+\mathrm{TF})(\mathrm{TN}=$ time exploring the novel object; $\mathrm{TF}=$ time exploring the familiar object). (C) Ketamine decreased total exploration time. (D) Schematic diagram for the social affiliation test. The focal rat is free to choose between a social target (stranger I, within a wire containment cup) and an object (empty cup). (E) Only the control group displayed the expected social preference significantly increasing the time spent in contact with the conspecific. (F) Schematic diagram of social novelty preference, a novel rat is added (stranger II) and the focal rat is free to investigate. (G) Control rats spent significantly more time in contact with the novel rat. Rats were treated for 7 days with ketamine $30 \mathrm{mg} / \mathrm{kg}$, IP starting on PND 35 and tested on PND60, controls received isovolumetric saline. $\mathrm{N}=5$ for both groups. Results were expressed as mean \pm SEM. $* P<0.05,{ }^{*} P<0.01$ compared with the control group (as determined by two-way ANOVA followed by the Sidak's post-hoc test, or two-tailed unpaired t-test for NOR).

and psychosis are predominant features in adolescents and young adults using ketamine for recreational purposes [38]. In accordance, the participants of a study using subanesthetic doses of ketamine in humans (I.V., four days) reported self-rated increased anxiety [39], which reinforces the relevance of evaluating anxiety in translational ketamine models.

Locomotor sensitization due to glutamate antagonists is usually only observable soon after ketamine dosing, or subsequently to a challenge with another NMDAR blocker $[12,20,23,40]$. Our data are in accordance with former studies where no changes in total locomotion were observed 24 or $48 \mathrm{~h}$ after the last administration of ketamine [21,23]. While we did not observe a difference in the total distance travelled by both groups, we found decreased activity in the central area, which is also indicative of increased anxiety and corroborates the EPM 
results.

Impairment of working memory has previously been reported in NMDAR antagonist models, but the extension of the resultant deficits appears to be greatly dependent on the nature of the pharmacological approach $[41,42,43]$. The NOR test has consistently been used as a first screening for working memory [17] and significant deficits were reported for ketamine, MK-801 or PCP [43]. Our data showed that chronic exposure to ketamine in adolescent rats, under the present administration schedule, did not affect the ability to discriminate the new object. Nevertheless, the time spent exploring the new object was reduced in the ketamine group. Two other studies using a similar exposure in adolescent rats also reported no effect of chronic ketamine in working memory [20,22]. Impaired memory under NMDAR antagonists was associated with NMDAR function in the prefrontal and hippocampal regions [44,45]. In mice exposed to ketamine on PND7, 9 and 11 impaired performance in the novel-object recognition and social novelty tests was associated to reduced parvalbuminexpressing cells in the medial PFC [46]. As such, we evaluated also the expression levels of several key markers for the glutamatergic and GABAergic function and found no evidence of glutamatergic hypofunction or decreased parvalbumin, which may justify the NOR results. Dopamine depletion in the prefrontal cortex was also shown to impair retention of familiar objects and motivation to explore [47]. Although we did not measure the levels of dopamine and its metabolites, we found decreased TH mRNA expression levels in the PFC of ketamine-exposed rats, which may contribute to decreased dopamine synthesis and lead to reduce exploration. In response to reduced dopamine, NMDAR antagonists were also reported to increase D1R frontal availability in chronic users [48]. However, in the present study we found no evidence of increased mRNA expression for dopamine receptors.

Deficits in social interaction and social recognition are associated with impaired social cognition and have been widely used to evaluate the action of NMDAR antagonists [43]. Our ketamine-exposed rats failed both to show significantly decreased contact time with the empty enclosure and significantly increased contact time with the unfamiliar rat. Reduced contact with a novel conspecific may be associated with depressive- and/or anxiety-like behaviors, which would be in accordance with our EPM results. This is also in accordance with previous studies using chronic $25 \mathrm{mg} / \mathrm{kg}$ of ketamine in adolescent rats, which reported decreased social contact associated to ketamine [20]. In adult rats, however, administration of ketamine (30 $\mathrm{mg} / \mathrm{kg}, 5$ consecutive days) did not affect social contact [49], which confirms the relevance of independently addressing the adolescent period.

Among the wide range of molecular dysfunctions reported for NMDAR dysfunction, calcium signalling seems to be a unifying element [50]. Post-synaptic density scaffolding proteins (mainly PSD-95) have been extensively implicated in dynamically modulating calcium signalling in synaptic development [50], plasticity [51] and stabilization of NMDAR [52]. It was also shown that changes in glutamatergic transmission, as those observed in in schizophrenia, might be both post-synaptic and presynaptic [53]. A critical component of synaptic plasticity in prefrontal presynaptic terminals is VGLUT1 [54]. Under the present administration schedule, we found no effect of ketamine in the expression levels of PSD95 and VGLUT1. Of note, these markers seem to vary substantially with the type of protocol used [55], which limits their predictive validity.

\section{Conclusion}

In summary, we report here that repeated exposure to ketamine in adolescent rats lead to increased anxiety as demonstrated by the EPM, open field and social contact results, but did not impact on working memory or novelty preference. In accordance, the present administration schedule did not affect glutamatergic e GABAergic markers typically associated with frontal dysfunction, such as parvalbumin, PSD95 a GAD67 levels. In contrast, TH expression was found reduced, which may be accompanied by decreased dopamine synthesis and therefore involved in the anxiety profile observed. Altogether, the present results also indicate that repeated adolescent ketamine may be a valuable preclinical model for anxiety.

Conflict of interest: The authors declare that they have no conflict of interest.

\section{References}

[1] J Frohlich and J. D. Van Horn, Reviewing the ketamine model for schizophrenia. J Psychopharmacol, 28 (2014), 287-302.

[2] B. D. Cohen, G. Rosenbaum, E. D. Luby, and J. S. Gottlieb, Comparison of phencyclidine hydrochloride (Sernyl) with other drugs. Simulation of schizophrenic performance with phencyclidine hydrochloride (Sernyl), lysergic acid diethylamide (LSD-25), and amobarbital (Amytal) sodium; II. Symbolic and sequential thinking. Arch Gen Psychiatry, 6(1962), 395-401.

[3] C. A. Jones, D, J. Watson, and K. C. Fone, Animal models of schizophrenia. Br J Pharmacol, 164(2011), 1162-1194.

[4] F. Sams-Dodd, Phencyclidine-induced stereotyped behaviour and social isolation in rats: a possible animal model of schizophrenia. Behav Pharmacol, 7(1996), 3-23.

[5] T. A. Jenkins, M. K. Harte, and G. P. Reynolds, Effect of subchronic phencyclidine administration on sucrose preference and hippocampal parvalbumin immunoreactivity in the rat. Neurosci Lett, 471(2010), 144-147.

[6] J. D. Jentsch, J. D. Elsworth, J. R. Taylor, D. E. Redmond, and R. H. Jr., Roth, Dysregulation of mesoprefrontal dopamine neurons induced by acute and repeated phencyclidine administration in the nonhuman primate: implications for schizophrenia. Adv Pharmacol, 42(1998), 810-814.

[7] R. Murai, Y. Noda, K. Matsui, H. Kamei, and A. Mouri, et al. Hypofunctional glutamatergic neurotransmission in the prefrontal cortex is involved in the emotional deficit induced by 
A

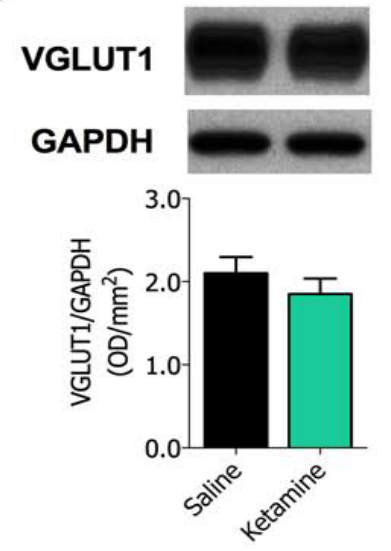

D
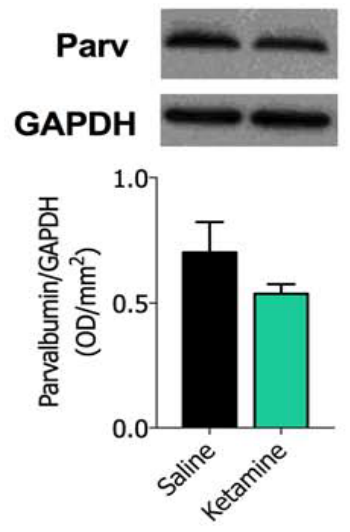

B
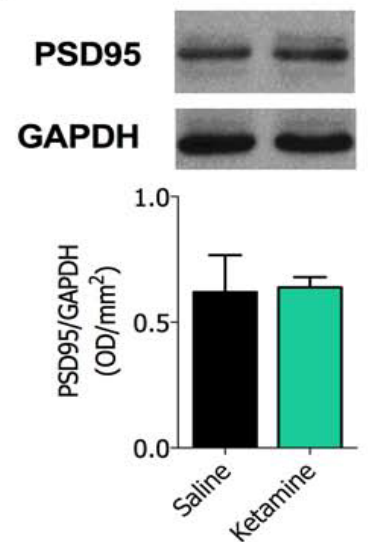

E
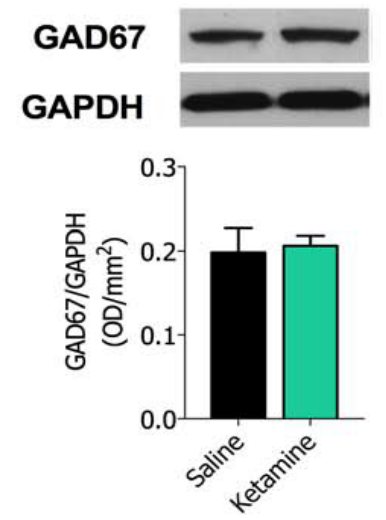

C
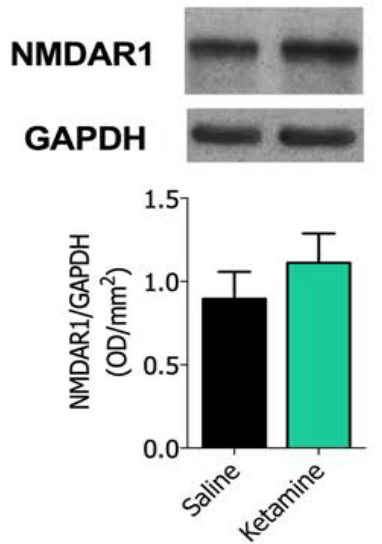

G

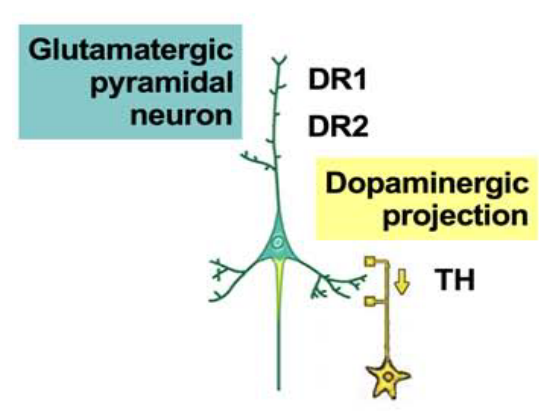

H

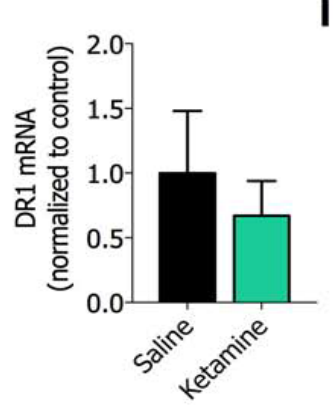

I

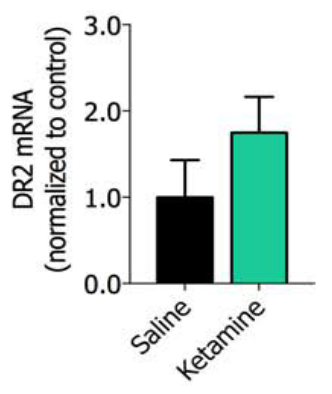

J

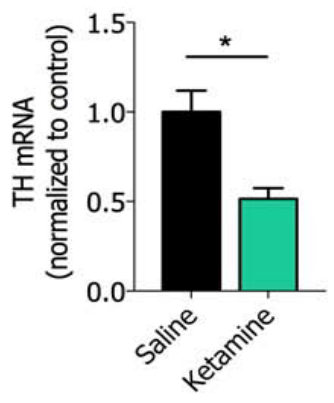

Figure 3: Ketamine affected dopaminergic but not glutamatergic and GABAergic markers. The protein expression levels of the (A) glutamate transporter VGLUT1, (B) post-synaptic density scaffolding protein (PSD95) and (C) the glutamate ionotropic receptor NMDAR1 were assessed by Western blot in protein extracts obtained from the prefrontal cortex of rats treated with saline solution or with ketamine $(30 \mathrm{mg} / \mathrm{kg}$, IP, for 7 days, starting on PND 35 and sacrificed after behavioral testing). The levels of (D) Parvalbumin and (E) glutamic acid decarboxylase (GAD67) were also assessed. (F) Schematic representation of the glutamatergic/GABAergic interaction at the prefrontal level, showing molecular players usually affected in schizophrenic patients. No differences were seen between ketamine and control rats for any of the tested markers. Data were normalized to the loading control GAPDH. Results are expressed as mean \pm SEM for 5 animals per group. (G) Schematic representation of the dopaminergic terminal at the prefrontal levels, showing molecular players usually affected in schizophrenic patients. The mRNA expression levels of (H) dopamine D1-like receptors (D1R), (I) dopamine D2-like receptors (D2R) and (J) tyrosine-hydroxylase (TH) were assessed in the PFC of rats treated with saline solution and with ketamine. No differences were seen between ketamine and control rats for D1R and D2R, while TH was significantly reduced in the ketamine-exposed group, which is compatible with a hypodopaminergic frontal state. Results were expressed as mean \pm SEM, $N=4$ animals per group. Values displayed were normalized to the control group. $* P<0.05$ compared with control group (as determined by two-tailed unpaired t-test). 
repeated treatment with phencyclidine in mice: implications for abnormalities of glutamate release and NMDA-CaMKII signaling. Behav Brain Res, 180(2007), 152-160.

[8] Z. Abdul-Monim, J. C. Neill, and G. P. Reynolds, Sub-chronic psychotomimetic phencyclidine induces deficits in reversal learning and alterations in parvalbumin-immunoreactive expression in the rat. J Psychopharmacol, 21(2007), 198-205.

[9] Y. K. Choi, S. Snigdha, M. Shahid, J. C. Neill, and F. I. Tarazi, Subchronic effects of phencyclidine on dopamine and serotonin receptors: implications for schizophrenia. J Mol Neurosci, 38(2009), 227-235.

[10] R. J. Beninger, T. W. Baker, M. M. Florczynski, and T. J. Banasikowski, Regional differences in the action of antipsychotic drugs: implications for cognitive effects in schizophrenic patients. Neurotox Res, 18(2010), 229-243.

[11] G. Keilhoff, A. Becker, G. Grecksch, G. Wolf, and H. G. Bernstein, Repeated application of ketamine to rats induces changes in the hippocampal expression of parvalbumin, neuronal nitric oxide synthase and cFOS similar to those found in human schizophrenia. Neuroscience, 126(2004), 591-598.

[12] A. Becker, B. Peters, H. Schroeder, T. Mann, and G. Huether, Ketamine-induced changes in rat behaviour: A possible animal model of schizophrenia. Prog Neuropsychopharmacol Biol Psychiatry, 27(2003), 687-700.

[13] S. Y. Kim, H. Lee, H. J. Kim, E. Bang, and S. H. Lee, In vivo and ex vivo evidence for ketamine-induced hyperglutamatergic activity in the cerebral cortex of the rat: Potential relevance to schizophrenia. NMR Biomed, 24(2011), 1235-1242.

[14] K. Kittelberger, E. E. Hur, S. Sazegar, V. Keshavan, and B. Kocsis, Comparison of the effects of acute and chronic administration of ketamine on hippocampal oscillations: relevance for the NMDA receptor hypofunction model of schizophrenia. Brain Struct Funct, 217(2012), 395-409.

[15] C. Hjorthoj, N. Albert, and M. Nordentoft, Association of Substance Use Disorders with Conversion From Schizotypal Disorder to Schizophrenia. JAMA Psychiatry (2018).

[16] L. Chen, A. Selvendra, A. Stewart, D. Castle, Risk factors in early and late onset schizophrenia. Compr Psychiatry, 80(2018), 155-162.

[17] B. K. Yee and P. Singer, A conceptual and practical guide to the behavioural evaluation of animal models of the symptomatology and therapy of schizophrenia. Cell Tissue Res, 354(2013), 221246

[18] C. E. Girardi, N. C. Zanta, and D. Suchecki, Neonatal stressinduced affective changes in adolescent Wistar rats: early signs of schizophrenia-like behavior. Front Behav Neurosci, 8(2014), 319.

[19] C. J. Alves, A. Magalhaes, P. R. Monteiro, and T. Summavielle, Very Long-Term Effects of Chronic Cocaine on Anxiety and Stress. In: Preedy VR, editor. The neuroscience of cocaine. Academic Press ed. London: Elsevier. pp. (2017) 774.

[20] C. S. Gama, L. Canever, B. Panizzutti, C. Gubert, and L. Stertz, Effects of omega-3 dietary supplement in prevention of positive, negative and cognitive symptoms: a study in adolescent rats with ketamine-induced model of schizophrenia. Schizophr Res, 141(2012), 162-167.

[21] J. Wang, M. Zhou, X. Wang, X. Yang, and M. Wang, Impact of ketamine on learning and memory function, neuronal apoptosis and its potential association with miR-214 and PTEN in adolescent rats. PLoS One, 9(2014), e99855.

[22] E. Ram, S. Raphaeli, and A. Avital, Prepubertal chronic stress and ketamine administration to rats as a neurodevelopmental model of schizophrenia symptomatology. Int $\mathrm{J}$ Neuropsychopharmacol, 16(2013), 2307-2314.

[23] E. M. Parise, L. F. Alcantara, B. L. Warren, K. N. Wright, and R. Hadad, Repeated ketamine exposure induces an enduring resilient phenotype in adolescent and adult rats. Biol Psychiatry, 74(2013), 750-759.
[24] K. J. Schoepfer, C. E. Strong, S. K. Saland, K. N. Wright, and M. Kabbaj, Sex- and dose-dependent abuse liability of repeated subanesthetic ketamine in rats. Physiol Behav, (2017).

[25] D. D'Souza and M. Sadananda, Estrous Cycle Phase-Dependent Changes in Anxiety- and Depression-Like Profiles in the Late Adolescent Wistar-Kyoto Rat. Ann Neurosci, 24(2017), 136-145.

[26] S. L. Andersen, Trajectories of brain development: point of vulnerability or window of opportunity? Neurosci Biobehav Rev, 27(2003), 3-18.

[27] A. C. Meirsman, A. Robe, A. de Kerchove d'Exaerde, and B L. Kieffer, GPR88 in A2AR Neurons Enhances Anxiety-Like Behaviors. eNeuro 3(2016).

[28] R. A. Bevins and J. Besheer, Object recognition in rats and mice: a one-trial non-matching-to-sample learning task to study 'recognition memory'. Nat Protoc, 1(2006), 1306-1311.

[29] A. Ennaceur, S. Michalikova, A. Bradford, and S. Ahmed, Detailed analysis of the behavior of Lister and Wistar rats in anxiety, object recognition and object location tasks. Behav Brain Res, 159(2005), 247-266.

[30] O. Kaidanovich-Beilin, T. Lipina, I. Vukobradovic, J. Roder, and J. R. Woodgett, Assessment of social interaction behaviors. J Vis Exp, (2011).

[31] N. Dawson, B. J. Morris, and J. A. Ratt, Subanaesthetic ketamine treatment alters prefrontal cortex connectivity with thalamus and ascending subcortical systems. Schizophr Bull, 39(2013), 366-377.

[32] F. Mueller, F. Musso, M. London, P. de Boer, and N. Zacharias, Pharmacological fMRI: Effects of subanesthetic ketamine on resting-state functional connectivity in the default mode network, salience network, dorsal attention network and executive control network. Neuroimage Clin, 19(2018), 745-757.

[33] F. Faul, E. Erdfelder, A. G. Lang, and A. Buchner, G*Power 3: a flexible statistical power analysis program for the social, behavioral, and biomedical sciences. Behav Res Methods, 39(2007), 175-191.

[34] D. A. Lewis, Neuroplasticity of excitatory and inhibitory cortical circuits in schizophrenia. Dialogues Clin Neurosci, 11(2009), 269-280.

[35] R. J. Rodgers, J. C. Cole, K. Aboualfa, and L. H. Stephenson, Ethopharmacological analysis of the effects of putative 'anxiogenic' agents in the mouse elevated plus-maze. Pharmacol Biochem Behav, 52(1995), 805-813.

[36] K. Wedzony, K. Fijal, M. Mackowiak, A. Chocyk, and W. Zajaczkowski, Impact of postnatal blockade of N-methylD-aspartate receptors on rat behavior: a search for a new developmental model of schizophrenia. Neuroscience, 153(2008), 1370-1379.

[37] S. J. Ballaz, H. Akil, and S. J. Watson, Previous experience affects subsequent anxiety-like responses in rats bred for novelty seeking. Behav Neurosci, 121(2007), 1113-1118.

[38] S. S. Kalsi, D. M. Wood, and P. I. Dargan, The epidemiology and patterns of acute and chronic toxicity associated with recreational ketamine use. Emerg Health Threats J, 4(2011), 7107.

[39] J. H. Krystal, L. P. Karper, A. Bennett, D. C. D'Souza, and A. Abi-Dargham, Interactive effects of subanesthetic ketamine and subhypnotic lorazepam in humans. Psychopharmacology (Berl), 135(1998), 213-229.

[40] A. Rocha, N. Hart, and K. A. Trujillo, Differences between adolescents and adults in the acute effects of PCP and ketamine and in sensitization following intermittent administration. Pharmacol Biochem Behav, 157(2017), 24-34.

[41] R. P. Daya, J. K. Bhandari, P. A. Hui, Y. Tian, and T. Farncombe, Effects of MK-801 treatment across several pre-clinical analyses including a novel assessment of brain metabolic function utilizing PET and CT fused imaging in live rats. Neuropharmacology, 77(2014), 325-333 
[42] B. Grayson, N. F. Idris, and J. C. Neill, Atypical antipsychotics attenuate a sub-chronic PCP-induced cognitive deficit in the novel object recognition task in the rat. Behav Brain Res, 184(2007), 31-38.

[43] J. C. Neill, S. Barnes, S. Cook, B. Grayson, and N. F. Idris, Animal models of cognitive dysfunction and negative symptoms of schizophrenia: focus on NMDA receptor antagonism. Pharmacol Ther, 128(2010), 419-432.

[44] J. Aura and P. Riekkinen Jr. Blockade of NMDA receptors located at the dorsomedial prefrontal cortex impairs spatial working memory in rats. Neuroreport, 10(1999), 243-248.

[45] T. Yoshihara and Y. Ichitani, Hippocampal N-methyl-D-aspartate receptor-mediated encoding and retrieval processes in spatial working memory: delay-interposed radial maze performance in rats. Neuroscience, 129(2004), 1-10.

[46] V. Jeevakumar, C. Driskill, A. Paine, M. Sobhanian, and H. Vakil, Ketamine administration during the second postnatal week induces enduring schizophrenia-like behavioral symptoms and reduces parvalbumin expression in the medial prefrontal cortex of adult mice. Behav Brain Res, 282(2015), 165-175.

[47] O. Y. Chao, M. E. Pum, and J. P. Huston, The interaction between the dopaminergic forebrain projections and the medial prefrontal cortex is critical for memory of objects: implications for Parkinson's disease. Exp Neurol, 247(2013), 373-382.

[48] H. Tsukada, S. Nishiyama, D. Fukumoto, K. Sato, and T. Kakiuchi, Chronic NMDA antagonism impairs working memory, decreases extracellular dopamine, and increases DI receptor binding in prefrontal cortex of conscious monkeys. Neuropsychopharmacology, 30(2005), 1861-1869.

[49] A. Becker and G. Grecksch, Ketamine-induced changes in rat behaviour: a possible animal model of schizophrenia. Test of predictive validity. Prog Neuropsychopharmacol Biol Psychiatry, 28(2004), 1267-1277.

[50] M. S. Lidow, Calcium signaling dysfunction in schizophrenia: a unifying approach. Brain Res Brain Res Rev, 43(2003), 70-84.

[51] L. Foa and R. Gasperini, Developmental roles for Homer: more than just a pretty scaffold. J Neurochem, 108(2009), 1-10.

[52] J. F. Sturgill, P. Steiner, B. L. Czervionke, and B. L. Sabatini, Distinct domains within PSD-95 mediate synaptic incorporation, stabilization, and activity-dependent trafficking. J Neurosci 29(2009), 12845-12854.

[53] N. D. Halim, C. S. Weickert, B. W. McClintock, T. M. Hyde, and D. R. Weinberger, Presynaptic proteins in the prefrontal cortex of patients with schizophrenia and rats with abnormal prefrontal development. Mol Psychiatry, 8(2003), 797-810.

[54] S. Takamori, J. S. Rhee, C. Rosenmund, and R. Jahn, Identification of a vesicular glutamate transporter that defines a glutamatergic phenotype in neurons. Nature, 407(2000), 189-194.

[55] V. Micale, J. Kucerova, and A. Sulcova, Leading compounds for the validation of animal models of psychopathology. Cell Tissue Res, 354(2013), 309-330. 
